\title{
LA DIRECCIÓN DEL PROCESO EDUCATIVO Y LAEVALUACIÓN DEL APRENDIZAJE
}

\author{
Olga Lidia Pérez González \\ Universidad de Camagüey, Cuba
}

\section{INTRODUCCIÓN}

Las Universidades son las instituciones llamadas a formar recursos humanos altamente calificados, que como soldados, deben luchar para poner a sus países en la senda del desarrollo. Hoy más que nunca la Universidad debe cumplir su misión histórica de mantener, trasmitir y desarrollar la cultura universal. "Universidad para el fortalecimiento de la libertad, de la dignidad y de la democracia"1.

Sin embargo, aún se manifiestan la existencia de problemas en el proceso educativo en la Educación Superior, en este sentido el Ministro de Educación de Cuba, Dr. Luis I. Gómez Gutiérrez, en el encuentro por la Unidad de los Educadores Latinoamericanos (Pedagogía 97), al referirse al desarrollo de la educación planteó: "Es necesario continuar profundizando en los colectivos de profesores sobre todo en el primer curso de las carreras, en los procedimientos de carácter pedagógico y profesional que fomentan hábitos correctos de estudio propio de cada nivel escolar a fin de que los estudiantes aprovechen mejor su autopreparación y cuenten con una base sólida para su educación continua una vez graduados".

En relación, a lo expresado por el Ministro, se observa que existen serias deficiencias en el proceso educativo en los primeros años de las carreras universitarias que atentan contra la formación del estudiante, entre otros: baja solidez de los conocimientos, poca incidencia de los estudiantes en la conducción del proceso educativo, poca dedicación de los estudiantes a la actividad de estudio, dificultades en la orientación para la autopreparación de las diferentes asignaturas, entre otras.

Estas deficiencias se revelan mucho más en las asignaturas básicas debido al nivel de profundidad y de asimilación del contenido que exigen los objetivos de estas asignaturas, dado que ellas aportan las leyes de la naturaleza que el hombre empleará para transformarla ${ }^{2}$ y máxime en la Matemática por ser ella misma básica en las Ciencias Naturales.

Se han realizado diferentes investigaciones a nivel internacional y nacional con el fin de dar solución a las deficiencias detectadas, varios trabajos evidencian estos esfuerzos, los cuales han sido dirigidos en varias direcciones. Ejemplo de ellos son los referidos al perfeccionamiento de la enseñanza de la matemática en la Educación Superior ${ }^{3},{ }^{4},{ }^{5}, 6$, a la orientación de las acciones de los estudiantes en el proceso de asimilación ${ }^{7}$, la determinación de la efectividad del uso de los ciclos temáticos ${ }^{8}$, el estudio de la concepción sistémico dialéctica a través de invariantes ${ }^{9}$, la formación de la actividad cognoscitiva de los estudiantes ${ }^{10}$ y el estudio de los procedimientos lógicos de la identificación y clasificación en estudiantes de Ciencias Técnicas ${ }^{11}$, entre otros. Lo más sobresaliente de estas investigaciones es que han resuelto el problema pero no con la eficiencia esperada. 
Vázquez ${ }^{12}$ en su investigación detectó en la primera etapa experimental que si no se organiza la autopreparación del estudiante los resultados son bajos, incluso propone que el libro de texto se redacte con la lógica de la organización del proceso e incluya la autopreparación. Esto nos indica que la variable autopreparación ha influenciado en los resultados de estas investigaciones y se observa que es más la insuficiencia que la deficiente autopreparación, o sea, hay falta de estudio por parte de los estudiantes, sin embargo la autora realizó una investigación documental encontrando que este problema ha estado latente hasta la fecha y que la dirección del proceso educativo ha sido deficiente pues en la generalidad de los casos no han existido mas sanciones que el reprobado, contribuyendo así a convertir el proceso en una lucha por aprobar, mas que por aprender.

Por otro lado la profesora Sánchez ${ }^{13}$ realizó una investigación con maestros que estaban estudiando Licenciatura en Educación y algunos especialistas en la enseñanza del idioma en Venezuela y detectó serias deficiencias idiomáticas y sin embargo esos estudiantes se titularon, como muchos profesionales titulados que trabajan como si fuesen técnicos medios, pues no tienen las habilidades necesarias para desarrollar la profesión. Villarroel al respecto plantea ${ }^{14}$ que son evaluaciones no válidas que permiten a un analfabeto funcional tener éxito en la carrera universitaria, lo que nos indica que el control como función de la dirección es deficiente.

La falta de estudio por parte de los estudiantes puede tener su origen en diferentes causas, pero en el centro de ellas está la deficiente dirección del proceso educativo, ya que generalmente sólo se afirma que los estudiantes no estudian, pero no hay acciones directas sobre ellos para erradicar el problema.

En la historia de las universidades, este fenómeno ha representado un problema que no ha tenido solución en el trabajo metodológico, ni ha sido resuelto en las investigaciones científicas, aunque si se ha estudiado este problema pero generalmente estos estudios van más a lo espitemológico y a lo teórico que a lo metodológico, siendo una constante preocupación, sobre todo en los primeros años de las carreras de ingeniería. Por lo que consideramos como problema científico a tratar en el presente trabajo: La deficiente dirección del proceso educativo, la cual propicia la falta de estudio por los estudiantes provocando la disminución del rendimiento académico y su calidad.

El objetivo del trabajo está en caracterizar el sistema de dirección del proceso docente educativo relevando a la evaluación del aprendizaje como una de sus funciones esenciales.

\section{DESARROLLO}

En la dirección del proceso, el profesor determina el orden de sus actividades, que incluye como sus funciones: la planificación, la organización, gerencia y la evaluación, estas funciones hacen que este proceso se desarrolle cíclicamente. Cada una de ellas tiene un carácter específico y pueden estudiarse independientemente, pero por su esencia, todas ellas están estrechamente interrelacionadas y se compenetran unas con las otras.

Por tanto, el proceso de dirección debe considerarse como un proceso único e integral y la delimitación de cada una de estas funciones sólo tiene como objetivo, el elaborar los métodos y procedimientos para la realización más efectiva en la práctica.

* La dirección del proceso docente se caracteriza como un sistema porque tiene: 
1) Cualidad resultante, que son los objetivos planteados.

2) Composición, que son los elementos requeridos: organización, planificación, gerencia y evaluación, para garantizar su funcionamiento.

3) Estructura, dada por el conjunto ordenado de relaciones entre dichos elementos para asegurar el cumplimiento de los objetivos. Esta estructura es:

- Estática cuando se ven las relaciones de estos componentes desde el punto de vista del espacio.

- Dinámica cuando se ven las relaciones de estos componentes en el tiempo.

4) Relación con el medio que lo rodea, ya que el mismo está expuesto a determinadas condiciones externas, a su contexto.

* Las características fundamentales de este sistema de dirección son las siguientes:

El isomorfismo, ya que su estructura es igual a la estructura del proceso educativo, por eso podemos decir que el sistema de dirección es isomorfo al proceso docente educativo como sistema.

La equifinalidad, que significa que en este sistema podemos obtener los mismos resultados finales a partir de diferentes condiciones iniciales y a través de una variedad de métodos, es decir, no siempre las características del colectivo estudiantil son las mismas y los métodos y medios utilizados pueden ser muy variados, pudiéndose obtener los mismos resultados.

La entropía, propiedad de este sistema, según la cual, la dirección del proceso educativo se va por encima de las características individuales y colectivas de los estudiantes y del proceso de asimilación de los contenidos, donde el estudiante apela a otros recursos, como lo es el fraude, y logra aprobar la asignatura, pero el carácter consciente de la asimilación de los conocimientos es deficiente. En este sentido se dice que el sistema no adquiere entropía, es decir, se puede culminar el proceso de forma exitosa desde el punto de vista docente, pero el aspecto educativo no se cumple, existiendo por tanto, dificultades en la formación de los estudiantes, afectando así la calidad de los resultados obtenidos, en fin, el sistema se sobrecarga y se da una ruptura entre la dirección de los aspectos docentes y educativos dándose una interacción negativa, pues el proceso continúa y los aspectos negativos no se logran superar.

La sinergia, que implica que el funcionamiento interrelacionado de todos los elementos del sistema permite obtener mejores resultados que los alcanzados por los elementos actuando aisladamente. En este sentido es importante que se logre un efecto sinérgico, lo que depende de la capacidad del profesor en la dirección del proceso de aprovechar el potencial de dichas interacciones.

Este sistema de dirección es vital para el desarrollo del proceso educativo y su función general es la de integrar las distintas partes y elementos de este proceso, así como su relación con el entorno, para lograr esto se deben llevar a cabo las funciones anteriormente mencionadas.

* La dirección del proceso educativo, sus funciones:

La planificación es la función mediante la cual se proyecta el desarrollo del proceso, por lo que implica la precisión de los objetivos, el contenido, el sistema de tareas a desarrollar, los problemas, los 
métodos y medios de enseñanza, para toda la asignatura y para cada una de las unidades que la componen.

En ella deben verse la combinación adecuada de las diferentes tareas para el desarrollo de habilidades y propiciar la asimilación consciente de los contenidos. Esta combinación se expresa como un conjunto particular de interrelaciones entre las diferentes tareas que dan lugar al desarrollo de la independencia cognoscitiva, sobre la base de las acciones esenciales de cada habilidad y de las habilidades más generales.

La organización tiene como objetivo establecer un orden interno coherente que permita el funcionamiento del proceso como una unidad, por lo que implica la estructuración y el ordenamiento interno de los componentes personales del proceso: profesor - alumno, y de los elementos del contenido de las diferentes materias (conocimiento, habilidades, hábitos y valores), con vista a lograr de la manera más eficiente los objetivos propuestos. Entonces, la organización del proceso supone dotar al mismo de una estructura que le permita coordinar e integrar el sistema de tareas planificado.

En esta organización coexisten la estructura formal e informal. La formal es la que se hace teniendo en cuenta el sistema de tareas planificadas desde el inicio del semestre, es decir las tareas previstas a desarrollar en clases. Mientras que la relación de las tareas no planificadas, y que surgen atendiendo a las diferencias individuales de los estudiantes, constituyen una organización informal.

Estas dos estructuras están en continua interrelación, lo que hace que generalmente sea imposible establecer la separación entre ambas, por tanto ellas coexisten y entre ambas se producen continuas interdependencias. Es necesario que la estructura formal sea sometida a las modificaciones oportunas, para así adaptarlas a las condiciones cambiantes del colectivo estudiantil, de cada estudiante y su entorno y que en el proceso de adaptación se puedan incorporar a la estructura formal aquellos elementos de la informal que sean considerados convenientes.

La gerencia del proceso educativo, se da a través de la ejecución del proceso en su dinámica y consiste en tomar decisiones para que el sistema se dirija en el sentido del cumplimiento de los objetivos.

La evaluación es el complemento lógico de la planificación y sus características dependen, y a la vez influyen, en la organización. Esta función es una de las esenciales de la dirección del proceso educativo el cual está sometido a las perturbaciones del entorno, y en consecuencia también de dicho proceso.

Su misión es lograr que el sistema se mantenga dentro de una trayectoria previamente definida, introduciendo las correcciones necesarias para evitar las desviaciones que se vayan produciendo, se trata, por tanto, lograr mantener la estructura del proceso y las interacciones entre los elementos de dicho sistema.

La evaluación debe estar dirigida al proceso de enseñanza y al de aprendizaje, lo que supone: el conocimiento de los aspectos didácticos y psicológicos que intervienen en el proceso, y la búsqueda de sinergias entre las diferentes actividades del mismo.

Este efecto sinérgico debe resultar en la interrelación y equilibrio de las siguientes regularidades:

- Evaluar el proceso de ascensión al objetivo y no sólo el objetivo.

- Evaluar teniendo en cuenta que el estado del estudiante puede cambiar. 
- Evaluación flexible estratégica, o sea, que exista la posibilidad siempre de mejorar una calificación.

- Evaluación transparente, para lograr un equilibrio en la valoración que hace el estudiante y la que hace el profesor.

- Evaluación de forma productiva.

- Evaluación de los objetivos educativos y constructivos.

- Evaluación dirigida a las particularidades de los alumnos.

- Evaluación de los procesos metacognoscitivos.

La variedad de la sinergia depende de las características de la materia que se imparte, de las características del profesional que se quiere formar, y otros aspectos.

Por ejemplo la sinergia del componente investigativo, se logra cuando se pueden aprovechar conjuntamente el trabajo investigativo con el desarrollo de una tarea específica, la cual conduce al conocimiento de la metodología de la investigación científica, al incremento de la actividad de estudio y asimilación consciente de los contenidos asociados a dicha tarea.

Cuanto más sea el efecto sinérgico de la evaluación, en la dirección del proceso educativo, más posibilidades de éxitos tendrá este.

La sinergia resultante a que conlleva la evaluación provoca, además, mayor interrelación de los diferentes componentes de la universidad como institución social: profesores, estudiantes, base material de estudio, dirección, etc.

La evaluación desde este marco que la presentamos, es cualitativa, en la que cada profesor debe ser un activo investigador, a través de la investigación - acción, como una necesidad definida por las propias exigencias del modelo del profesional que se quiere formar, lo cual exige:

- El estudio integral del estudiante.

- El análisis racional de las oportunidades y amenazas que se presentan, ya sea en relación a otras materias o en relación al propio colectivo estudiantil.

- El análisis racional de los puntos débiles y fuertes del colectivo estudiantil y de cada estudiante en particular.

Esto conlleva a una evaluación sistemática y rigurosa en busca de los aspectos positivos y negativos y de una compatibilidad entre ambos, o sea, la dirección del proceso, que lleva a cabo el profesor, debe enfatizar los puntos fuertes del colectivo estudiantil, mermando sus puntos débiles, permitirá salir al paso de las amenazas de su entorno y aprovechar las oportunidades que este ofrece.

Todo esto induce a ver la gerencia como interpretación de la evaluación, considerando a esta última como un proceso progresivo dentro del cual el profesor va obteniendo diversos indicadores sobre el proceso de asimilación de los estudiantes, donde cada uno de estos indicadores deben incidir en el proceso educativo, sobre la base de un efecto sinérgico favorable al proceso. 
Esta organización cualitativa de la evaluación del aprendizaje no rechaza la utilización de instrumentos (como técnicas cuantitativas), sino que se incluye la información producidas por estos instrumentos dentro de una lógica cualitativa, vinculada de forma indirecta con los indicadores diversos constituidos según la actividad valorativa de profesores y estudiantes.

Por tanto, no es minimizar lo cuantitativo, sino que lo cualitativo y lo cuantitativo se fundan en busca de una calidad superior, ocupando así el lugar que le corresponde, no como forma secundaria o complementaria en relación con lo cuantitativo.

En resumen la evaluación como una de las funciones de la dirección del proceso educativo se introduce como elemento cuya función principal es coordinar los distintos subsistemas en la dirección de manera que se puedan alcanzar los objetivos, teniendo en cuenta todas sus interrelaciones.

\section{CONCLUSIONES}

A pesar de que hemos clasificado cada una de las funciones en la dirección del proceso educativo, existe una constante interacción entre estas, por lo que, en la realidad su separación es prácticamente inexistente.

Es necesario en el sistema de dirección del proceso educativo la búsqueda de un equilibrio global, referido a la sistematización entre su concepción e implementación, entre los aspectos psicosociales, cognoscitivos y educativos. También es importante un equilibrio entre el enfoque y la metodología seguida para la dirección, en el cual la exigencia de sistematización de rigor y formalización debida a la complejidad del proceso, no debe implicar una eventual rigidez.

Luego, la búsqueda de la flexibilidad y capacidad de adaptación de la evaluación a las características del proceso y de la constante coherencia entre los diferentes elementos de la realidad del mismo, sólo se puede encauzar mediante un enfoque contingente.

El enfoque de contingencia en la evaluación se refiere a que no debemos suponer que existirá una evaluación mejor que otra, un instrumento mejor que otro, sino que esto depende del colectivo estudiantil, del profesor, del momento y circunstancia y de acuerdo a las condiciones en que se realiza el proceso, es decir un enfoque contingente de la evaluación debe recoger en su seno las exigencias de una adaptación rápida y activa del proceso educativo a la evolución del colectivo estudiantil.

De acuerdo con lo aquí estudiado, se infiere que la evaluación debe ir a lo que falta para el logro de los objetivos, y no a si estos se cumplen o no. Por tanto concluimos que La evaluación del aprendizaje es una de las funciones del sistema de dirección del proceso educativo mediante la cual el profesor, los alumnos y el colectivo estudiantil concientizan el grado de desarrollo de lo que han aprendido y de lo que les falta aún para la consecución de los objetivos de aprendizaje ${ }^{15}$.

\section{REFERENCIAS BIBLIOGRÀFICAS}

1 La transformación universitaria en vísperas del tercer milenio. Memorial del simposio. AUGM/SM/UDUAL.—Caracas: Editorial CRESALC/UNESCO/1996.—Pág. 7-10

2 GutiérReZ, A. M.; PortuOndo, P. R. : Dialéctica de los ciclos de los planes de estudio. Ponencia de la V Conferencia de Ciencias de la Educación. Camagüey. 1999 
3 Calderón ARIOSA. Regla : Perfeccionamiento de la enseñanza del cálculo integral en Ingeniería Mecánica. Informe de investigación. ISPJAE. Ciudad Habana. 1994.

4 HERNÁNDEZ FERNÁNDEZ, H. El perfeccionamiento de la enseñanza de las Matemáticas en la Educación Superior Cubana. Tesis de Grado. 1989

5 MARTínez RODRíGuez, F. Acerca de la Organización del proceso de asimilación del cálculo Diferencial. Tesis de Grado. 1991

6 Rodríguez HUNG., Teresa. Enfoque sistémico en la dirección de la asimilación de los conceptos básicos de la disciplina. Tesis de Doctor. Ciudad La Habana. 1991.

7 BLANCO, R. La orientación de las acciones del estudiantes en el proceso de asimilación. Revista Cubana de Educación Superior. Vol XVI, No 2/ 1994

8 BLANCO, R. La determinación de la Efectividad del uso de los ciclos temáticos. ISBN 959-16-001-3. Centro de Ediciones Electrónicas del MES. Cuba. 1997.

9 GaRCía BLANCO, R. Concepción sistémico - dialéctica de la teoría de invariantes. Convención Internacional de Eventos Universitarios. IV Taller sobre la Educación Superior y sus Perspectivas. Ciudad de la Habana. 1998.

10 TALízINA N., F. La formación de la actividad cognoscitiva de los estudiantes. México. Edit. Angeles. 1992.

11 Sanz CabreRA, T. Estudio de los procedimientos lógicos de identificación de conceptos y clasificación en estudiantes de ciencias técnicas. Tesis de grado. 1989.

12 VÁZQUEZ CEDEÑo, Rosa. La resolución de problemas y tareas docentes de matemática IV para Ingeniería Eléctrica. ". Tesis de Doctorado. Universidad de Camagüey. Cuba. Enero de 2000.

13 SÁNCHEZ, L. : La comprensión de la Lectura en los docentes que se profesionalizan a nivel superior. Edit. U.C.V., Caracas. Venezuela. 1998.

14 VILLARROEL, C.: La enseñanza Universitaria: de la transmisión del saber a la construcción del conocimiento. En revista Educación Superior y Sociedad; Vol. 6, No. 1, pág. 103-122, 1995.

15 PÉREZ GonzÁlEZ, Olga Lidia. "La evaluación del aprendizaje como elemento del sistema de dirección del proceso docente educativo en la enseñanza de las matemáticas para ciencia técnicas". Tesis de Doctorado. Universidad de Camagüey. Cuba. Enero de 2000. 


\title{
Contactar
}

Revista lberoamericana de Educación

\author{
Principal OEI
}

